\title{
Frequency-domain imaging algorithm for ultrasonic testing by application of matrix phased arrays
}

\author{
Dmitry Dolmatov ${ }^{1, *}$, Yana Salchak ${ }^{1}$, and Roman Pinchuk ${ }^{2}$ \\ ${ }^{1}$ Tomsk Polytechnic University, 634050 Tomsk, Russia \\ ${ }^{2}$ ACS-Solutions GmbH, Science Park 2, 66123 Saarbrücken, Germany
}

\begin{abstract}
Constantly increasing demand for high-performance materials and systems in aerospace industry requires advanced methods of nondestructive testing. One of the most promising methods is ultrasonic imaging by using matrix phased arrays. This technique allows to create three-dimensional ultrasonic imaging with high lateral resolution. Further progress in matrix phased array ultrasonic testing is determined by the development of fast imaging algorithms. In this article imaging algorithm based on frequency domain calculations is proposed. This approach is computationally efficient in comparison with time domain algorithms. Performance of the proposed algorithm was tested via computer simulations for planar specimen with flat bottom holes.
\end{abstract}

\section{Introduction}

Methods of nondestructive testing are used extensively in aerospace industry due to high requirements for materials and systems quality. Ultrasonic testing has become one of the most promising techniques for this purpose considering its accuracy and low costing [1].

Accuracy of the measurement can be influenced by the type of the chosen transducer. Ultrasonic phased array probes are most common for industrial applications. Due to the quantity of the elements it provides diversity of scanning laws that is important for the precise imaging. In general, phased array transducers can be divided into two groups: linear and matrix phased arrays. The main advantage of matrix phased array transducers is that it can be used for 3-D reconstruction. Application of linear arrays is limited for this purpose [2]. Further progress in ultrasonic imaging is determined by development of fast imaging algorithms within the application of matrix phased arrays.

There are two basic types of imaging algorithms: frequency-domain and time-domain. However, it is known that frequency-domain algorithms have better computational speed in comparison with time-domain type [3]. By that reason it is challenging to develop a new three-dimensional imaging algorithm based on calculations in frequency domain. In this article the algorithm based on so-called Stolt transformation is proposed [4]. The solution is formed in accordance with the results of the previous research for two-dimensional

\footnotetext{
* Corresponding author: dolmatovdo@tpu.ru
} 
geometry and single element case [5]. Thereby, frequency domain algorithm based on Stolt mapping should be extended for application of full matrix capture technique in order to build three-dimensional reconstruction.

\section{Materials and methods}

The proposed algorithm consists of three main steps. First is application of full matrix capture technique. Thus, the function is to be changed for separate transmitter and receiver elements. The second step is extrapolation of the wave field to the depth of the boundary between water and the specimen. And the final step is three-dimensional Stolt mapping of scattered data.

Full matrix capture is the technology that involves collecting of scattered data from the full set of receiver/transmitter pairs. In this case the measured wave field can be described by the function $F\left(t, x_{t r}, y_{t r}, z_{t r}, x_{r}, y_{r}, z_{r}\right)$. This function depends on time and coordinates of the transmitter and the receiver. In this article application of a plane matrix phased array is proposed. Due to this fact one of the coordinates of transmitter and receiver will be the same. In this case function $F\left(t, x_{t r}, y_{t r}, x_{r}, y_{r}, z\right)$ can be reconsidered. It is to be transformed to $F(t, x, y, z)$, where $\mathrm{x}$ and y variables are found according to the following correlations:

$$
\begin{aligned}
& x=\frac{x_{t r}+x_{r}}{2} \\
& y=\frac{y_{t r}+y_{r}}{2}
\end{aligned}
$$

The next step is wave field extrapolation to the boundary depth between water and the specimen. Firstly, it is necessary to calculate three-dimensional fast Fourier transform of the scattered data:

$$
P\left(\omega, k_{x}, k_{y}, z\right)=0.125 \pi^{-3} \iint_{-\infty}^{\infty} \int F(t, x, y, z) e^{-i\left(k_{x} x+k_{y} y-\omega t\right)} d x d y d t,
$$

where $k_{x}, k_{y}$ are wavenumbers, $\omega$ is a temporal frequency and $0.125 \pi^{-3}$ is normalization constant.

After that the wave field extrapolation to the desired depth is calculated:

$$
P\left(\omega, k_{x}, k_{y}, z+\Delta z\right)=P\left(\omega, k_{x}, k_{y}, z\right) e^{i k_{z} \Delta z} .
$$

The last step is Stolt mapping of extrapolated wave field. The final image of the specimen is derived according to the equation:

$$
I(x, y, z)=\iint_{-\infty}^{\infty} \int \frac{c_{l}}{2} \frac{k_{z}}{\sqrt{k_{x}^{2}+k_{y}^{2}+k_{z}^{2}}} P\left(k_{x}, k_{y}, k_{z}\right) e^{-i\left(k_{x} x+k_{y} y+k_{z} z\right)} d k_{x} d k_{y} d k_{z},
$$

where $c_{l}$ is a velocity of longitudinal waves in specimen. It needs to be noted that before the implementation of this equitation it is vital to change the variable. It means that temporal frequency $\omega$ is to be changed according to the wavenumber $k_{z}$ :

$$
\omega\left(k_{x}, k_{y}, k_{z}\right)=\frac{c_{l}}{2} \sqrt{k_{x}^{2}+k_{y}^{2}+k_{z}^{2}}
$$




\section{Results and discussion}

Computer simulation is used for verification of the proposed algorithm. This simulation is built using special software CIVA 2015. This is a powerful and versatile tool for computer simulations of ultrasonic inspections [6].

The simulated specimen is a planar steel block within several flat bottom holes. The diameter of each hole is $1 \mathrm{~mm}$. Simulation setup is shown in Figure 1. Each flaw is marked by a corresponding letter. The position of each hole can be determined by $\mathrm{x}$ and $\mathrm{y}$ coordinates. Further, each defect has its own particular depth. Hereby, the position is to be described by three parameters $(\mathrm{x}, \mathrm{y}, \mathrm{z})$, where $\mathrm{x}$ and $\mathrm{y}$ are coordinates of the flat bottom hole and $\mathrm{z}$ is its depth (all values are in $\mathrm{mm}$ ). In total there were five flat bottom holes created for simulation. The corresponding coordinates are: $\mathrm{A}(-5 ; 5 ; 35), \mathrm{B}(-2.5 ; 2.5 ; 30), \mathrm{C}(0 ; 0 ; 15)$, $\mathrm{D}(2.5 ;-2.5 ; 20), \mathrm{E}(5 ;-5 ; 25)$.
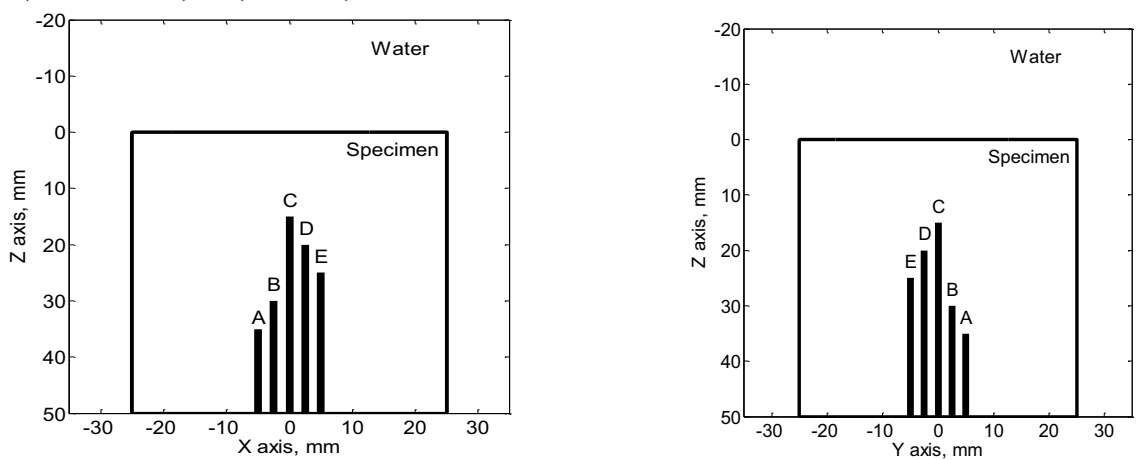

Fig. 1. Simulation setup.

In order to achieve reliable results, it is important to choose appropriate parametres of the transducer. A sixty four element phased matrix array was chosen as a transducer with the frequency of $5 \mathrm{MHz}$. Width and length of each element of the matrix is $0.5 \mathrm{~mm}$. Spacing between elements is $0.1 \mathrm{~mm}$. As for the inspection settings, the step of $2 \mathrm{~mm}$ was chosen for accurate simulation.

Proposed algorithm is post processing technique for imaging of received acoustic signals. Thus, the results of the simulation were used as input data. For the estimation of the proposed algorithm efficiency Matlab 2016a (The MathWorks, Natick, MA) was implemented.

A common way of three-dimensional ultrasonic imaging is visualization of the backscattered amplitude maximum corresponding to each measuring point along the depth. This approach was used to demonstrate the results of algorithm implementation given in Figure 2.
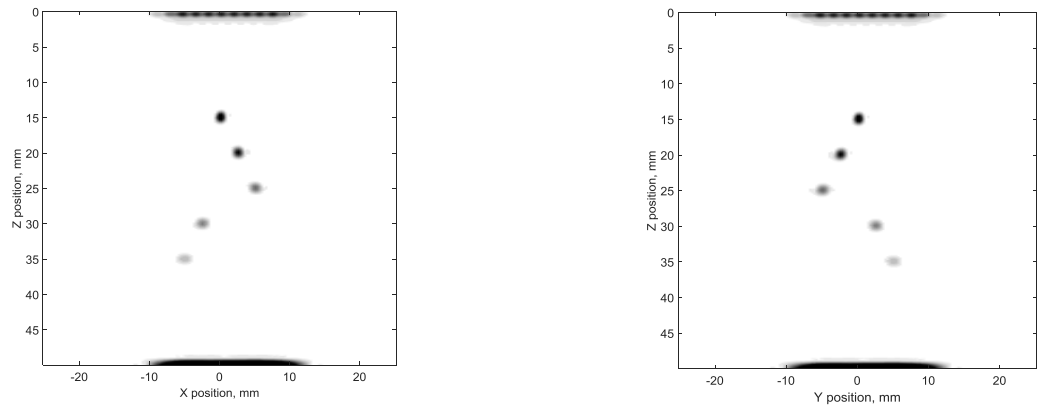

Fig. 2. The results of algorithm implementation. 
Experimental results demonstrate that the proposed frequency-domain algorithm was improved and can be applied for three-dimensional imaging within the matrix phased array application. Accurate and precise measurements results were received for each of the flaws by testing the suggested algorithm.

Another important parameter that can influence the reliability of imaging is positioning precision of the system. Robotic instruments are generally applied in order to achieve higher precision. However, the calibration of these systems is quite challenging. In our previous research we suggest a novel approach of noncontact calibration that can be implemented for this purpose $[7,8]$.

\section{Conclusions}

Computer simulation in CIVA was made to verify the proposed algorithm. This is a novel frequency-domain 3-D imaging algorithm for ultrasonic testing with matrix phased arrays. The achieved results are validated and suggest solution that can be applied for effective immersion ultrasonic testing of planar specimens within the application of matrix phased arrays.

The reported study was partially supported by the Governmental program "Science", research project No. 11.6342.2017/BCh.

\section{References}

1. G. Scott, C. M. Scala, NDT international 15.2, 75 (1982)

2. O. Oralkan, A. S. Ergun, C.H. Cheng, J.A. Johnson, M. Karaman, T,H. Lee, B.T. Khuri-Yakub, IEEE T Ultrason Ferr 50(11), 1581 (2003)

3. A.J. Hunter, B.W Drinkwater, P.D Wilcox, IEEE T Ultrason Ferr 55(11), 2450 (2008)

4. R.H Stolt, Geophysics 43.1, 23 (1978)

5. D. Dolmatov, V. Abramets, Matec Web Conf 48, 3004 (2016)

6. P. Calmon, S. Mahaut, S. Chatillon, R. Raillon, Ultrasonics 44, 975 (2006)

7. D. Dolmatov, V. Zhvyrblya, G. Filippov, Y. Salchak, E. Sedanova, IOP Conf Ser Mater Sci Eng 135(1), 12010 (2016)

8. G. Filippov, V. Zhvyrblya, S. Sharavina, Y. Salchak, Matec Web Conf 48, 3002 (2016) 